\title{
Multinodular and Vacuolating Posterior Fossa Lesions of Unknown Significance
}

\author{
(D) A. Lecler, (D). Bailleux, (D) B. Carsin, (D) H. Adle-Biassette, (D) B. Baloglu, (D) C. Bogey, (DF. Bonneville, (D)E. Calvier, (DP.-O. Comby, \\ (D).-P. Cottier, (D) F. Cotton, (D) Reschamps, (DC. Diard-Detoeuf, (D). Ducray, (D). Duron, (D). Drissi, (D) M. Elmaleh, (D). Farras, \\ (D).A. Garcia, (DE. Gerardin, (DS. Grand, (DD.C. Jianu, (D). Kremer, (D) N. Magne, (D) M. Mejdoubi, (D) A. Moulignier, (D) M. Ollivier, \\ (D) S. Nagi, (D). Rodallec, (D).-C. Sadik, (DN. Shor, (D). Tourdias, DC. Vandendries, (DV. Broquet, and (D). Savatovsky; \\ for the ENIGMA Investigation Group (EuropeaN Interdisciplinary Group for MVNT Analysis)
}

\begin{abstract}
SUMMARY: Multinodular and vacuolating neuronal tumor of the cerebrum is a rare supratentorial brain tumor described for the first time in 2013. Here, we report 11 cases of infratentorial lesions showing similar striking imaging features consisting of a cluster of low T1-weighted imaging and high T2-FLAIR signal intensity nodules, which we referred to as multinodular and vacuolating posterior fossa lesions of unknown significance. No relationship was found between the location of the lesion and clinical symptoms. A T2-FLAIR hypointense central dot sign was present in images of 9/11 (82\%) patients. Cortical involvement was present in 2/11 (18\%) of patients. Only 1 nodule of 1 multinodular and vacuolating posterior fossa lesion of unknown significance showed enhancement on postcontrast TIWI. DWI, SWI, MRS, and PWI showed no malignant pattern. Lesions did not change in size or signal during a median follow-up of 3 years, suggesting that multinodular and vacuolating posterior fossa lesions of unknown significance are benign malformative lesions that do not require surgical intervention or removal.
\end{abstract}

ABBREVIATIONS: IQR interquartile range; MVNT = multinodular and vacuolating neuronal tumor of the cerebrum; MV-PLUS = multinodular and vacuolating posterior fossa lesions of unknown significance

M ultinodular and vacuolating neuronal tumor of the cerebrum (MVNT) is a rare brain tumor described for the first time in 2013 and added in the World Health Organization Classification of Tumors of the Central Nervous System in 2016. ${ }^{1,2}$ Its prevalence and pathophysiology are unknown. It is of-

Received June 10, 2019; accepted after revision July 27.

From the Departments of Neuroradiology (A.L., J.B., L.D., J.-C.S., C.V., V.B., J.S.) and Neurology (R.D., A.M.), Fondation Ophtalmologique A. Rothschild, Paris, France; Department of Radiology (B.C., J.S.), Centre Hospitalier Régional Universitaire de Rennes, Rennes, France; Department of Pathology (H.A.-B.), Lariboisière Hospital, Paris Diderot, Paris-Cité-Sorbonne University, Paris, France; Department of Radiology (S.B., S.K.), University Hospital of Strasbourg, Strasbourg, France; Department of Neuroradiology (C.B.), Centre Hospitalier Universitaire Limoges, Limoges, France; Department of Neuroradiology (F.B.), Hôpital Pierre-Paul-Riquet, Centre Hospitalier Universitaire Purpan, Toulouse, France; Neurology Department (E.C., J.A.G.), Hôpital René et Guillaume-Laënnec, Centre Hospitalier Universitaire de Nantes, Saint-Herblain, France; Department of Vascular and Interventional Radiology (P.-O.C.), Image-Guided Therapy Center, François-Mitterrand University Hospital, Dijon Cedex, France; Department of Radiology (J.-P.C.), Centre Hospitalie Régional Universitaire de Tours, Tours, France; Brain and Imaging Laboratory (J.-P.C.), UMR U930, National Institute for Health and Medical Research, FrançoisRabelais University, Tours, France; Service de Radiologie (F.C.), Centre Hospitalier Lyon-Sud, Hospices Civils de Lyon, University Claude Bernard Lyon 1, Lyon, France Centre de Recherche en Acquisition et Traitement de l'Image pour la Santé (CREATIS) (F.C.), National Institute for Health and Medical Research U1044/Centre National de la Recherche Scientifique (CNRS) Unité Mixte de Recherche (UMR) 5220, Lyon, France; Department of Neurology (C.D.-D.), CH Sainte-Périne, Paris, France; Department of Neuro-Oncology (F.D.), Lyon French Reference Center of Paraneoplastic Neurological Syndrome, Hospices Civils de Lyon, Hôpital Neurologique, Bron Cedex, France; Synatac Team (F.D.), NeuroMyoGene Institut, National Institute for Health and Medical Research U1217/Centre National de la Recherche Scientifique (CNRS) Unité Mixte de Recherche (UMR) 5310, Lyon, France; University Claude Bernard Lyon 1 (F.D.), Lyon, France; Institut National de ten asymptomatic and discovered incidentally. It remains unclear whether MVNT should be considered a true neoplasm or a malformative lesion. ${ }^{1-7}$

MVNTs have been reported to show highly suggestive imaging features, especially with MR imaging. ${ }^{8-16}$ MVNT consists of the coalescence of small T2-weighted imaging and T2-FLAIR

Neurologie (C.D., S.N.), Service de Neuroradiologie, Université de Tunis El Manar, Faculté de Médecine de Tunis, Tunis, Tunisia; Pediatric Radiology Department (M.E.), Robert Debré Hospital, Paris, France; Jordi Radiologia C/de la Roda (J.F.), Andorra la Vella, Andorra; Department of Neuroradiology and MRI (E.G., N.M.) Rouen University Hospital, Rouen, France; Neuroradiologie Diagnostique et Interventionnelle et IRM Nord (S.G.), Centre Hospitalier et Universitaire de Alpes Grenoble, Grenoble, France; Department of Neurology (D.C.J.), Victor Babes University of Medicine and Pharmacy, Timisoara, Romania; Department of Neuroradiology (M.M.), University Hospital of Martinique, Fort-de-France, Martinique, France; Groupe Hospitalier Pellegrin (M.O.), Bordeaux, France; Clinique les Berges du Lac (S.N.), les Berges du Lac, Tunis, Tunisia; Centre d'Imagerie Centre Cardiologique du Nord (M.R.), CCN, Saint-Denis, France; Department of Neuroradiology (N.S.), Pitié-Salpêtrière Hospital, Paris, France; Service de Neuroimagerie Diagnostique et Thérapeutique (T.T.), Centre Hospitalier Universitaire de Bordeaux et National Institute for Health and Medical Research U1215, Université de Bordeaux, Bordeaux, France; Centre d'Imagerie Médicale Paris 15ème (C.V.), RMX, Paris, France; Department of Neuroradiology (V.B.), Centre Hospitalier Universitaire Lille, Lille, France; and Imagerie Paris 13 (J.S.), Paris, France. Please address correspondence to Augustin Lecler, MD, PhD, Department of Neuroradiology, Fondation Ophtalmologique Adolphe de Rothschild, 25 rue Manin, 75019 Paris, France; e-mail: alecler@for.paris; @RothNeuroRad

Indicates article with supplemental on-line table.

Indicates article with supplemental on-line photo.

http://dx.doi.org/10.3174/ajnr.A6223 
hyperintense nodules in subcortical and juxtacortical areas, with rare or no postcontrast enhancement. It is considered a "leaveme-alone" lesion because of the absence of malignancy criteria and the lack of evolutivity on follow-up MRIs. ${ }^{8,9}$

All MVNTs reported so far in the literature involved the supratentorial part of the brain. We report 11 patients from 24 international centers with lesions exhibiting a remarkably similar pattern of imaging findings in the posterior fossa, which we will refer to in this article as multinodular and vacuolating posterior fossa lesions of unknown significance (MV-PLUS).

The aim of our study was to describe the MR imaging characteristics at diagnosis and during follow-up.

\section{CASE SERIES}

\section{Study Design}

We conducted a multicenter retrospective study in 24 international centers specializing in neurologic diseases. This study was approved by our institutional Research Ethics Board (Fondation Ophtalmologique A.Rothschild) and adhered to the tenets of the Declaration of Helsinki. This study follows the Strengthening Reporting of Observational Studies in Epidemiology (STROBE) guidelines.

\section{Patients}

From July 2014 to February 2019, seventy-four patients with a suspicion of MVNT were identified and collected from the PACS of 24 international centers. Inclusion criteria were the following: 1) the presence of a lesion suggestive of MVNT on MR imaging, defined as a subcortical or juxtacortical lesion consisting of clusters of discrete or confluent high T2-FLAIR signal intensity small nodules; 2) the absence of an obvious differential diagnosis such as asymmetric enlargement of perivascular spaces, defined as smoothly demarcated fluid-filled cysts showing the same attenuation or intensity as CSF; cortical dysplasia, defined as a cortical thickening with general blurring at the white matter-gray matter junction; or a low-grade glial lesion defined as a cortically centered rounded mass with regular margins, with or without postcontrast enhancement; and 3) with a minimum patient follow-up of 12 months.

Among them, 12/74 (16\%) were located in the posterior fossa, thus, they could not be strictly considered MVNT because MVNT refers, by definition, to lesions involving the cerebrum. One of these 12 patients had an associated brain stem glioblastoma and was excluded. The remaining 11 patients were included for analysis. Their lesions will be referred to as MV-PLUS throughout this article.

\section{Clinical Charts}

All patients' medical charts were systematically reviewed. Demographic features were recorded as well as symptoms prompting imaging and clinical reports, including a comprehensive neurologic examination and follow-up. The presence of a relationship between the location of MV-PLUS and extension and clinical symptoms was evaluated and assessed as follows: no relationship, possible relationship, or definite relationship.

\section{MR Imaging}

All MR imaging examinations were performed on 3T scanners with a 16- or a 32-channel head coil. The minimal common protocol included multiplanar or 3D-T1- and T2-FLAIR-weighted imaging. Depending on the centers, postcontrast T1WI, T2WI, high-resolution T2WI, DWI, T2*WI or SWI. PWI and proton MRS were acquired in $3(27 \%)$ patients. A single-voxel pointresolved proton spectroscopy sequence with a short TE of $35 \mathrm{~ms}$ was used for all 3 patients. A single-voxel point-resolved proton spectroscopy sequence with a long TE of $135 \mathrm{~ms}$ was used for 2 patients. Voxel placement included both the lesion and normal tissue with a ratio of approximately $80 \% / 20 \%$, respectively.

\section{Image Analysis}

MR imaging examinations were anonymized and sent to a single core laboratory for reading. Three readers, 1 radiologist with 1 year of experience who had previously studied MVNT extensively prior this study and had acquired substantial expertise in MVNT (V.B.) and 2 neuroradiologists with 4 and 9 years of experience respectively (J.B. and A.L.) reviewed all MRIs in a consensus analysis. All reading sessions were completed on a dedicated workstation using Horos software (Nimble Co, Annapolis, Maryland).

The readers assessed the following characteristics of MVPLUS at diagnosis and during follow-up:

- Precise locations in the posterior fossa, divided into the 6 following areas: vermis, left or right cerebellar hemisphere, left or right cerebellar peduncle, and brain stem. The center of the lesion and all areas involved were reported separately.

- Overall size defined by the longest diameter of the lesion on T2-FLAIR. The size of the nodules of each lesion was reported as well.

- Signal intensity on T1-, T2-, and T2-FLAIR-weighted imaging. Signal was compared with that of the normal-appearing cerebellar white matter.

- The presence of enhancement on postcontrast T1WI.

- The presence of a restriction of the diffusion on DWI.

- The presence of intratumoral susceptibility signal or a blooming on $\mathrm{T}^{*}$ or SWI.

- Measurement of the relative CBV and relative CBF on PWI. Two ROIs were drawn: the first one inside the largest nodule of a MV-PLUS, the second one in a healthy cerebellar white matter region. Both ROIs were equal in size.

- Quantification of MRS metabolites at 2 TEs (35 and $135 \mathrm{~ms}$ ). We calculated 3 indices: choline/creatine, choline/ $N$-acetyl aspartate, and $\mathrm{N}$-acetyl aspartate/creatine.

- The presence of a T2-FLAIR central dot sign, defined as a T2FLAIR hypointense punctiform signal at the center of at least 1 hyperintense nodule.

- The type of margins between the nodules and the adjacent normal-appearing white matter, defined as sharp or blurred.

- The presence of cortical involvement.

- The presence of a mass effect, defined as any shift in any of the intracranial structures, including ventricles.

- Associated imaging abnormalities.

The readers assessed the presence of a change in the size or the signal of MV-PLUS during follow-up. The size was defined 
by the longest diameter of the lesion on FLAIR. 3D reformatting and coregistration were performed for readers to measure the lesion in the exact same plane as in the first MR imaging. A size change was defined as positive in the case of a change superior to or equal to $5 \%$ of initial size. A signal change was defined as positive in the case of a change of at least 1 component of the signal of the lesion on any of the sequences used, compared with the normal-appearing cerebellar white matter.

\section{Statistical Analysis}

Analyses were conducted using $\mathrm{R}$ software, Version 3.3.2. ${ }^{17}$ Categoric data were reported as a number (percentage) as

\section{Table 1: Patient characteristics and clinical data}

\begin{tabular}{lcc}
\hline & $\begin{array}{c}\text { No. of } \\
\text { Patients } \\
(\boldsymbol{n}=11)\end{array}$ & $\begin{array}{c}\text { Percentage } \\
(\%)\end{array}$ \\
\hline Sex & 5 & 45 \\
$\quad$ Male & 6 & 55 \\
$\quad$ Female & $37 \pm 13$ & \\
Age (mean) (yr) & & \\
Clinical symptoms prompting & & \\
$\quad$ initial imaging & 4 & 36 \\
$\quad$ Headache & 2 & 18 \\
$\quad$ Meningioma screening & 1 & 9 \\
$\quad$ Bilateral upper arm & & \\
$\quad$ paresthesia & 1 & 9 \\
$\quad$ Hearing loss & 1 & 9 \\
$\quad$ Tinnitus & 1 & 9 \\
$\quad$ Dizziness & 1 & 9 \\
\hline Aneurysm screening & & \\
\hline
\end{tabular}

appropriate. Continuous data were reported as median with interquartile range or mean $\pm \mathrm{SD}$ as appropriate.

\section{RESULTS}

\section{Patient Characteristics and Clinical Data}

Eleven patients were included (6 women and 5 men; mean age, $37 \pm 13$ years; range, $22-70$ years). Headache was the most frequent symptom prompting initial imaging. No relationship was found between the location of MV-PLUS and clinical symptoms. Median follow-up was 3.6 years (interquartile range $[\mathrm{IQR}]=1$ year), No patient developed symptoms potentially related to the MV-PLUS. No patient underwent an operation during follow-up. Detailed patient characteristics are presented in Table 1 .

\section{MV-PLUS MR Imaging Characteristics at Diagnosis}

Lesions were all located in the cerebellum. No lesion involved the brain stem. Almost all lesions centered on the vermis extended laterally to the cerebellar hemispheres: 5/7 (71\%). Conversely, none of the lesions centered on a cerebellar hemisphere extended to the vermis (Fig 1).

Lesions were all hyperintense on T2-FLAIR and T2WI and all hypointense on T1WI except for 1. One patient presented with postcontrast enhancement of the nodule of 1 lesion (Fig 2). A central dot sign was visible in $9 / 11(82 \%)$ patients (Online Figure). Cortical involvement was present in $2 / 11$ (18\%) patients. Detailed MR imaging characteristics are presented in Table 2 and the On-line Table.
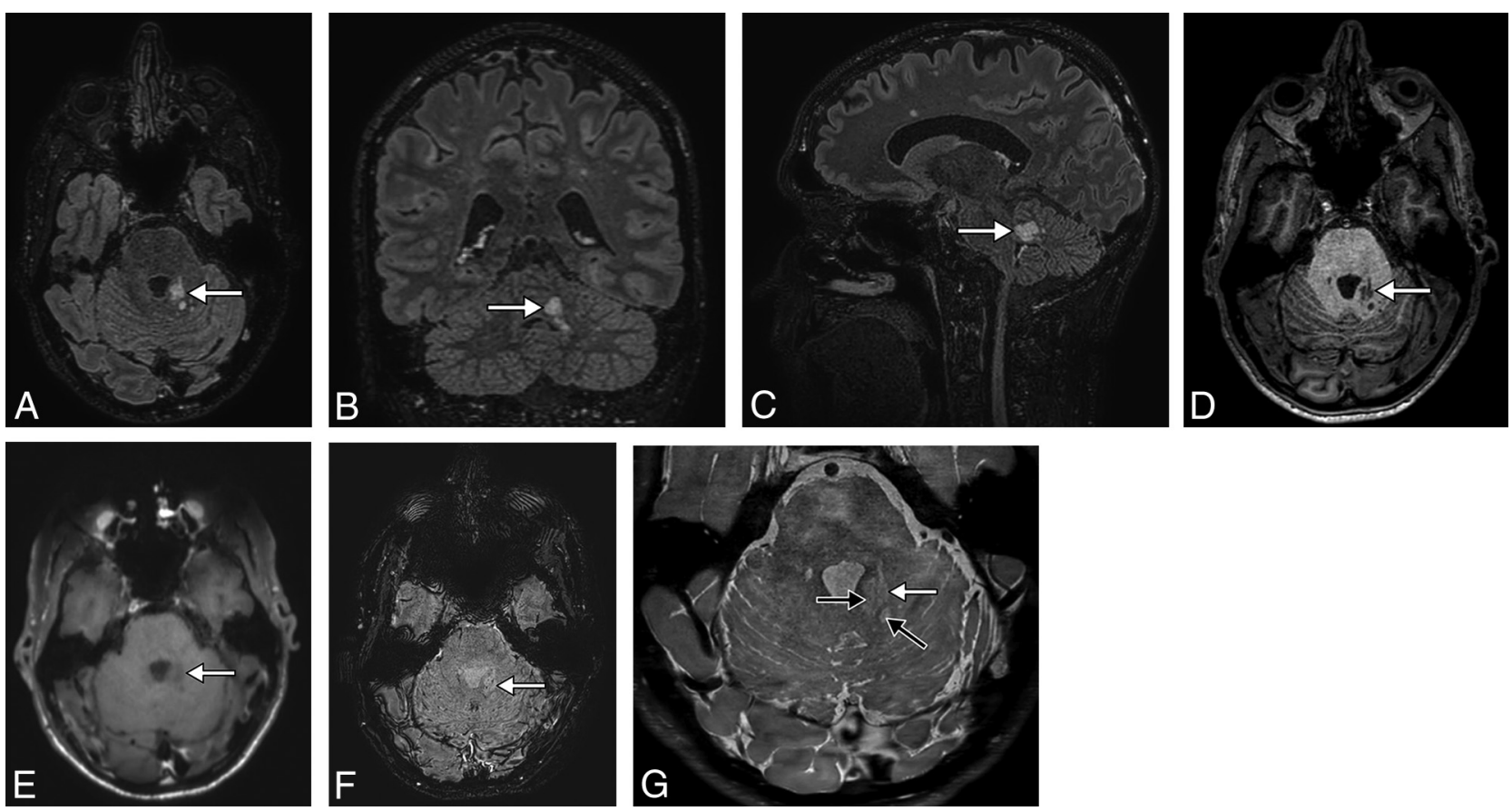

FIG 1. A 38-year-old man presenting with headache. 3D-T2-FLAIR reformatted in the axial (A), coronal (B), and sagittal (C) planes shows a high-signal intensity lesion (white arrow) of the posterior part of the left cerebellar peduncle, consisting of a coalescence of small nodules, highly suggestive of MV-PLUS. The lesion is hypointense on axial TIWI (D) and does not enhance on postcontrast TIWI (E). SWI $(F)$ shows no blooming or intratumoral susceptibility signal. High-resolution T2WI (G) shows hypointensity in the center of hyperintense nodules (black arrows), consistent with a central dot sign. Note the small mass effect and distortion of the lateral margin of the fourth ventricle. 

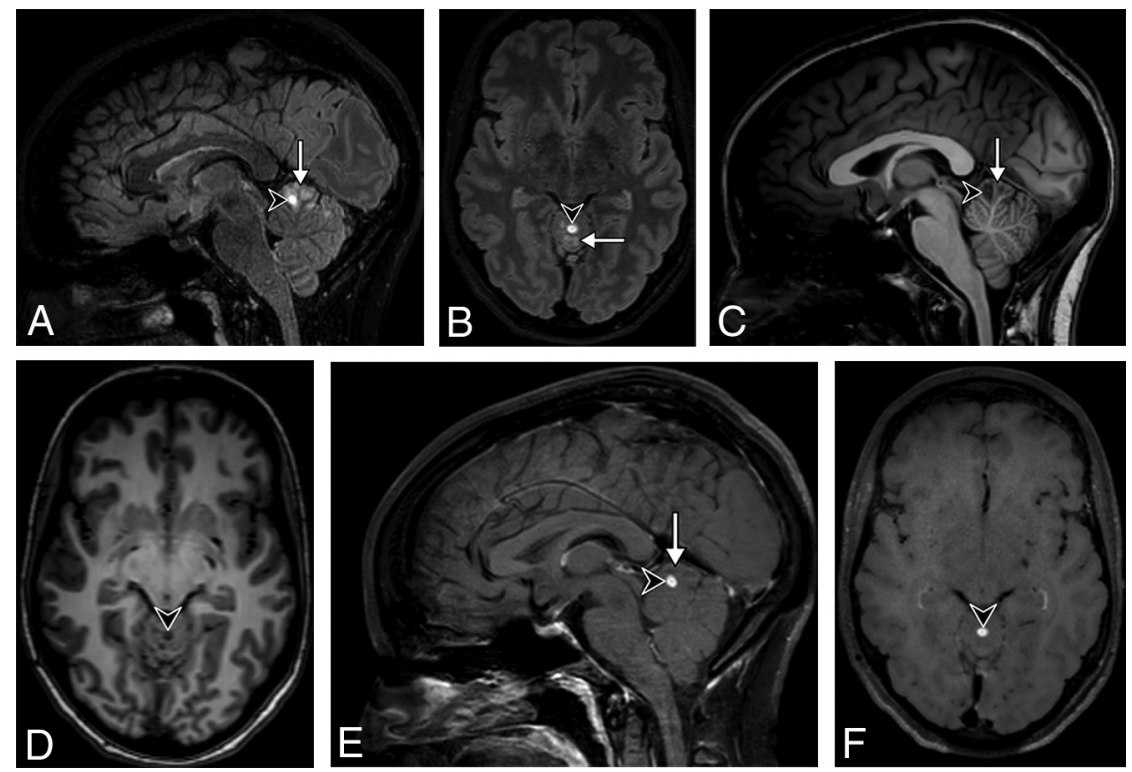

FIG 2. A 31-year-old woman presenting with headache. 3D-T2-FLAIR reformatted in the sagittal $(A)$ and axial (B) planes shows a high signal intensity multinodular lesion (arrow) of the upper vermis, highly suggestive of an MV-PLUS. Almost all clustered nodules are hypointense on 3D-TTWI reformatted in the sagittal $(C)$ and axial $(D)$ planes and do not enhance on postcontrast 3D-TTWI reformatted in the sagittal $(E)$ and axial $(F)$ planes. One anterior nodule (arrowhead) shows a substantially higher T2-FLAIR and lower T1 signal intensity than all the others, with a marked enhancement after contrast injection. Note the T2-FLAIR hypointense central dot sign does not enhance on postcontrast TIWI.

\section{MV-PLUS MR Imaging Characteristics during Follow-Up}

The median follow-up was 3.1 years (IQR $=1$ year). None of the described lesions changed size or signal during follow-up.

\section{DISCUSSION}

We describe the imaging characteristics of a new entity that we referred to as MV-PLUS. This entity has never been described in the literature to the best of our knowledge.

MV-PLUS imaging features consist of the coalescence of small T1WI hypointense and T2-FLAIR hyperintense nodules in subcortical and juxtacortical areas. They are very similar to those described in MVNT, a rare and recently described brain tumor, ${ }^{8-14}$ which was added in the World Health Organization Classification of Tumors of the Central Nervous System in 2016. ${ }^{1,2}$ None of the case reports and small series published in the literature so far have reported posterior fossa MVNT. Among 74 patients analyzed in 24 international centers showing MR imaging features highly suggestive of MVNT, we found only $11(15 \%)$ posterior fossa lesions, suggesting that MV-PLUS might be 10 times rarer than MVNT.

Similar to MVNT, MV-PLUS showed no sign of malignancy on MR imaging and an absence of evolutivity during followup. ${ }^{8,9}$ In our study, MR imaging showed neither restriction on DWI nor intratumoral susceptibility signal on T2* or SWI. PWI showed no increase in relative CBF or CBV. MRS showed no increase in choline peaks. There was no or only a small mass effect on the surrounding posterior fossa structures. Moreover, no changes in size or signal could be observed during follow-up, and no relationship was observed between the location of lesions and clinical symptoms. Only 1 nodule in 1 patient showed enhancement after contrast injection, which is similar to the low rate of enhancement reported for MVNT in the literature. ${ }^{8,9}$ However, this nodule had a very distinct signal compared with other nodules throughout all sequences. Enhancing MVNTs were reported in the literature to show only faint enhancement, suggesting that it might be a distinct lesion. ${ }^{8,9}$

Differential diagnoses for posterior fossa intra-axial lesions encompass a wide range of diseases, such as ischemic lesions, inflammatory diseases like multiple sclerosis, infectious diseases, vascular malformations, neoplastic lesions, degenerative lesions like ataxias, toxic lesions, malformative lesions like dysplastic cerebellar gangliocytoma, or normal variants like enlargement of perivascular spaces. ${ }^{18-20}$ However, both the location in juxtacortical or subcortical regions and typical features such as the presence of clusters of discrete or confluent high T2-FLAIR signal intensity small nodules make the diagnosis of MV-PLUS very likely. Moreover, we reported the presence of a T2-FLAIR hypointense central dot sign for most MV-PLUS lesions. This central dot sign was present in most nodules of $>4 \mathrm{~mm}$ and was more conspicuous on high-resolution T2WI. It was missing in images of 2 patients with low-resolution imaging. This central dot sign was already reported in the literature in MVNT, but its prevalence is not known. ${ }^{8,10}$ It might reflect the presence of a high protein or a solid component within the vacuolated areas, which is a typical pathology feature reported in MVNT histopathologic studies. ${ }^{1,7}$ It might be an interesting imaging criterion that could increase readers' confidence when diagnosing MV-PLUS. This sort of evidence might help rule out differential diagnoses.

Two patients had very subtle cortical involvement on imaging, which has not been reported in MVNT. 8 ,9 However, these 2 patients had low-resolution images, with 2D-FLAIR images with a section thickness of 3 and $5 \mathrm{~mm}$, respectively. Distinguishing cortical and subcortical areas is more challenging in the posterior fossa than in supratentorial regions. Thus, this appearance might be due to partial averaging rather than true cortical involvement and should be confirmed by other observations with high-resolution imaging.

MV-PLUS should probably be considered a leave-me-alone lesion, requiring no operation to confirm the diagnosis. Its nature remains unknown, and it is not clear whether it should be considered a neoplasm or a malformation. However, the absence of changes during follow-up, the absence of or only a small mass effect or malignant pattern on MR imaging, and the absence of a relationship with clinical symptoms might suggest that MV- 
Table 2: MR imaging characteristics of multinodular and vacuolating posterior fossa lesions of unknown significance (MV-PLUS) at diagnosis and during follow-up

No. of Patients

Percentage (\%)

\begin{tabular}{|c|c|c|}
\hline \multicolumn{3}{|l|}{ Location } \\
\hline \multicolumn{3}{|l|}{ Center of the lesion } \\
\hline \multicolumn{3}{|l|}{ Cerebellum } \\
\hline Vermis & $7 / 11$ & 64 \\
\hline Left cerebellar hemisphere & $1 / 11$ & 9 \\
\hline Right cerebellar hemisphere & $2 / 11$ & 18 \\
\hline Left cerebellar peduncle & $1 / 11$ & 9 \\
\hline Right cerebellar peduncle & $0 / 11$ & 0 \\
\hline Brain stem & $0 / 11$ & 0 \\
\hline \multicolumn{3}{|l|}{ All areas involved } \\
\hline \multicolumn{3}{|l|}{ Cerebellum } \\
\hline Vermis & $7 / 11$ & 64 \\
\hline Left cerebellar hemisphere & $3 / 11$ & 27 \\
\hline Right cerebellar hemisphere & $8 / 11$ & 73 \\
\hline Left cerebellar peduncle & $1 / 11$ & 9 \\
\hline Right cerebellar peduncle & $0 / 11$ & 0 \\
\hline Brain stem & $0 / 11$ & 0 \\
\hline Overall size (median) (IQR) (mm) & $27(24)$ & \\
\hline Size of the nodules (median) (IQR) (mm) & $4(5)$ & \\
\hline \multicolumn{3}{|l|}{ DWI } \\
\hline Restriction & $0 / 11$ & 0 \\
\hline \multicolumn{3}{|l|}{ T2* or SWI } \\
\hline Presence of ITSS & $0 / 11$ & 0 \\
\hline Blooming & $0 / 11$ & 0 \\
\hline \multicolumn{3}{|l|}{ PWI } \\
\hline rCBV (median) (IQR) & $0.62(0.06)$ & \\
\hline rCBF (median) (IQR) & $0.61(0.06)$ & \\
\hline \multicolumn{3}{|l|}{ MRS } \\
\hline \multicolumn{3}{|l|}{$\mathrm{TE}=35 \mathrm{~ms}$} \\
\hline Choline/creatine (median) (IQR) & $0.9(0.2)$ & \\
\hline Choline/N-acetyl aspartate (median) (IQR) & $0.9(0.1)$ & \\
\hline $\mathrm{N}$-acetyl aspartate/creatine (median) (IQR) & $1(0.2)$ & \\
\hline \multicolumn{3}{|l|}{$\mathrm{TE}=135 \mathrm{~ms}$} \\
\hline Choline/creatine (median) (IQR) & $1.2(0)$ & \\
\hline Choline/N-acetyl aspartate (median) (IQR) & $1(0)$ & \\
\hline $\mathrm{N}$-acetyl aspartate/creatine (median) (IQR) & $1.2(0)$ & \\
\hline \multicolumn{3}{|l|}{ Type of margins } \\
\hline Sharp & $11 / 11$ & 100 \\
\hline Blurred & $0 / 11$ & 0 \\
\hline \multicolumn{3}{|l|}{ Mass effect } \\
\hline Yes & $4 / 11$ & 36 \\
\hline No & $7 / 11$ & 64 \\
\hline \multicolumn{3}{|l|}{ Associated imaging abnormalities } \\
\hline None & $7 / 11$ & 64 \\
\hline Small-vessel disease & $3 / 11$ & 27 \\
\hline Colloid cyst & $1 / 11$ & 9 \\
\hline Cholesteatoma & $1 / 11$ & 9 \\
\hline
\end{tabular}

Note:-ITSS indicates intratumoral susceptibility signal; $\mathrm{rCBV}$, relative $\mathrm{CBV}$; rCBF, relative $\mathrm{CBF}$.

than expected and might be due to a selection bias. The first patient had a sibling recently diagnosed with a meningioma. The second patient had been taking cyproterone acetate for a long time, which had been reported to increase the chance of developing a meningioma.

Second, 2 centers contributed $>1$ case. Most of the 24 centers where clinicians were aware of MVNT had not identified any patients with similar imaging findings, suggesting that MVPLUS might be very rare.

Third, none of our patients underwent an operation; thus, we do not have any histopathologic evidence showing that MV-PLUS might be, in fact, MVNT. The posterior fossa is a very challenging region for surgery, and none of our patients had clinical symptoms related to their lesions; thus, none of them became surgical candidates. This is why we chose to remain cautious and to refer to this entity as MV-PLUS instead of posterior fossa MVNT. Given the benign MR imaging characteristics of MVPLUS and the absence of evolutivity with time, it might be extremely difficult to obtain pathologic details in the near future.

Fourth, the examinations were performed on various MR imaging devices from different vendors. Protocols were heterogeneous among the centers, mixing comprehensive multiparametric protocols and basic lowresolution MR imaging protocols. Therefore, our analysis of MV-PLUS might not be optimal. Some of the information we provide, such as the absence of a central dot sign or the involvement of the cortex, might

PLUS is a benign malformative lesion rather than a true neoplasm. However, we advise clinicians to perform a comprehensive protocol when characterizing possible MV-PLUS, including thin-millimetric T2- or T2-FLAIR sequences to detect the central dot sign, and MR spectroscopy as well as PWI to show the absence of malignant criteria. We also suggest long-term follow-up to assess any changes in size or signal.

Our study has some limitations: First, although we analyzed patients from 24 international centers, the results of the study are somewhat limited by the relatively small number of patients. Two asymptomatic patients underwent MR imaging for meningioma screening, representing $18 \%$ of our population, which is higher be inaccurate because of low-resolution images in 2 patients. PWI and MRS were performed in only 3/11 (27\%) patients; thus, the quantitative values we provided might be inaccurate. Moreover, only single-voxel spectroscopy was performed, which might have resulted in volume averaging with normal tissue.

\section{CONCLUSIONS}

We provided the first description of a new entity that we referred to as MV-PLUS. Our observation might help clinicians diagnose this entity, adapting their patient management and possibly avoiding an operation.

AJNR Am J Neuroradiol 40:1689-94 Oct 2019 www.ajnr.org 
Disclosures: Fabrice Bonneville-UNRELATED: Employment: Année. Elisabeth Auffrey-Calvier-UNRELATED: Board Membership: optimizing the MRI practices of patient with Sclérose en Plaques (SEP) in the Hôpitaux Universitaires Grand Ouest (HUGO) inter-region; Consultancy: place of imaging in the differential diagnosis of neurologic pathologies, focus on multiple sclerosis. Roman Deschamps-UNRELATED: Support for Travel to Meetings for the Study or Other Purposes: Biogen. Julien Savatovsky-UNRELATED: Payment for Lectures Including Service on Speakers Bureaus: Novartis, Biogen, Medtronic, Philips Healthcare, Sanofi; Travel/Accommodations/Meeting Expenses Unrelated to Activities Listed: Bayer. Stéphane Kremer-UNRELATED: Board Membership: Bayer; Payment for Lectures Including Service on Speakers Bureaus: Bayer Healthcare, Novartis, Biogen, Roche; Travel/Accommodations/Meeting Expenses Unrelated to Activities Listed: Biogen.

\section{ACKNOWLEDGMENT}

Laura McMaster provided professional English-language medical editing of this article.

\section{REFERENCES}

1. Huse JT, Edgar M, Halliday J, et al. Multinodular and vacuolating neuronal tumors of the cerebrum: 10 cases of a distinctive seizureassociated lesion: multinodular and vacuolating neuronal tumors. Brain Pathol 2013;23:515-24 CrossRef Medline

2. Louis DN, Perry A, Reifenberger G, et al. The 2016 World Health Organization Classification of Tumors of the Central Nervous System: a summary. Acta Neuropathol 2016;131:803-20 CrossRef Medline

3. Choi E, Kim SI, Won JK, et al. Clinicopathological and molecular analysis of multinodular and vacuolating neuronal tumors of the cerebrum. Hum Pathol 2019;86:203-12 CrossRef Medline

4. Calderon-Garcidueñas AL, Mathon B, Lévy P, et al. New clinicopathological associations and histoprognostic markers in ILAE types of hippocampal sclerosis. Brain Pathol 2018;28:644-55 CrossRef Medline

5. Pekmezci M, Stevers M, Phillips JJ, et al. Multinodular and vacuolating neuronal tumor of the cerebrum is a clonal neoplasm defined by genetic alterations that activate the MAP kinase signaling pathway. Acta Neuropathol 2018;135:485-88 CrossRef Medline

6. Thom M, Liu J, Bongaarts A, et al. Multinodular and vacuolating neuronal tumors in epilepsy: dysplasia or neoplasia? Brain Pathol 2018;28:155-71 CrossRef Medline

7. Yamaguchi M, Komori T, Nakata Y, et al. Multinodular and vacuolating neuronal tumor affecting amygdala and hippocampus: a quasi-tumor? Pathol Int 2016;66:34-41 CrossRef Medline
8. Nunes RH, Hsu CC, da Rocha AJ, et al. Multinodular and vacuolating neuronal tumor of the cerebrum: a new "leave-me-alone" lesion with a characteristic imaging pattern. AJNR Am J Neuroradiol 2017;38:1899-904 CrossRef Medline

9. Alsufayan R, Alcaide-Leon P, de Tilly LN, et al. Natural history of lesions with the MR imaging appearance of multinodular and vacuolating neuronal tumor. Neuroradiology 2017;59:873-83 CrossRef Medline

10. Badat N, Savatovsky J, Charbonneau F, et al. Multinodular vacuolating and neuronal tumor of the cerebrum. Neurology 2017;89:30405 CrossRef Medline

11. Shitara S, Tokime T, Akiyama Y. Multinodular and vacuolating neuronal tumor: A case report and literature review. Surg Neurol Int 2018;9:63. CrossRef Medline

12. Gonzalez-Quarante LH, Ruiz-Juretschke F, Sola Vendrell E, et al. Multinodular and vacuolating neuronal tumor of the cerebrum: a rare entity-new case and review of the literature. Neurocirugia (Astur) 2018;29:44-55 CrossRef Medline

13. Makrakis D, Veneris S, Papadaki E. Multinodular and vacuolating neuronal tumor incidentally discovered in a young man: conventional and advanced MRI features. Radiol Case Rep 2018;13:960-64 CrossRef Medline

14. Fukushima S, Yoshida A, Narita Y, et al. Multinodular and vacuolating neuronal tumor of the cerebrum. Brain Tumor Pathol 2015; 32:131-36 CrossRef Medline

15. Monté A-S, D’Arco F, De Cocker L. Multinodular and vacuolating neuronal tumor in an adolescent with Klinefelter syndrome. Neuroradiology 2017;59:1187-88 CrossRef Medline

16. Johnson DR, Guerin JB, Giannini C, et al. 2016 updates to the WHO Brain Tumor Classification System: what the radiologist needs to know. Radiographics 2017;37:2164-80 CrossRef Medline

17. The R Project for Statistical Computing, 2019. https://www.Rproject.org. Accessed March 11, 2019

18. Loevner LA. Imaging features of posterior fossa neoplasms in children and adults. Semin Roentgenol 1999;34:84-101 CrossRef Medline

19. Gass A, Filippi M, Grossman RI. The contribution of MRI in the differential diagnosis of posterior fossa damage. J Neurol Sci 2000;172:(Suppl 1):S43-S49 CrossRef Medline

20. Shih Y-Y, De La Garza BH, Huang S, et al. Comparison of retinal and cerebral blood flow between continuous arterial spin labeling MRI and fluorescent microsphere techniques. J Magn Reson Imaging 2014;40:609-15 CrossRef Medline 